\title{
Neurosurgical education in Europe and the United States of America
}

\author{
Jan-Karl Burkhardt, \\ Department of Neurosurgery, University Hospital, University of Zurich, Frauenklinikstr 10, Nord 1 \\ 8091 Zurich, Switzerland. Regenerative Medicine Program, Department of Surgical Research, \\ University Hospital Zurich, Zurich, Switzerland
}

Pascal O. Zinn,

Department of Neurosurgery, Beth Israel Deaconess Medical Center, Harvard Medical School, Boston, MA, USA. Department of Radiation Oncology, Division of Genomic Stability and DNA

Repair, Dana-Farber Cancer Institute, Harvard Medical School, Boston, MA, USA

Oliver Bozinov, Department of Neurosurgery, University Hospital, University of Zurich, Frauenklinikstr 10, Nord 1 8091 Zurich, Switzerland

Rivka R. Colen,

Department of Radiology, Brigham and Women's Hospital, 75 Francis Street, Boston, MA, USA

Helmut Bertalanffy, and

Department of Neurosurgery, University Hospital, University of Zurich, Frauenklinikstr 10, Nord 1 8091 Zurich, Switzerland

Ekkehard M. Kasper

Department of Neurosurgery, Beth Israel Deaconess Medical Center, Harvard Medical School, Boston, MA, USA

Jan-Karl Burkhardt: JanKarl.Burkhardt@gmail.com

\section{Abstract}

Training in neurological surgery is one of the most competitive and demanding specializations in medicine. It therefore demands careful planning in both the scientific and clinical neurosurgery arena to finally turn out physicians that can be clinically sound and scientifically competitive. National and international training and career options are pointed out, based on the available relevant literature, with the objective of comparing the neurosurgical training in Europe and the USA. Despite clear European Association of Neurosurgical Societies guidelines, every country in Europe maintains its own board requirements, which is reflected in an institutional curriculum that is specific to the professional society of that particular country. In contrast, the residency program in the USA is required to comply with the Accreditation Council for Graduate Medical Education guidelines. Rather similar guidelines exist for the education of neurosurgical residents in the USA and Europe; their translation into the practical hospital setting and the resulting clinical lifestyle of a resident diverges enormously. Since neurosurgical education remains heterogeneous worldwide, we argue that a more standardized curriculum across different nations would greatly facilitate the interaction of different centers, allow a direct comparison of available services, and support the 
exchange of vital information for quality control and future improvements. Furthermore, the exchange of residents between different training centers may improve education by increasing their knowledge base, both technically as well as intellectually.

\section{Keywords}

Neurosurgery; Education; Residency; Curriculum; United States; Europe

\section{Introduction}

Both Europe and the USA have a long tradition in biomedical education in neurosurgery [15]. It was Dr. W. Halsted in the early twentieth century who introduced the format of a residency program to America as a tool to improve clinical practice. As a surgeon teaching at Johns Hopkins Medical School in Baltimore, his aim was to guarantee a well-organized training period for young medical doctors to finally become qualified surgeons-and this model is still considered the standard for education in any medical specialization [14]. However, a vast range of different neurosurgical education programs have developed worldwide ever since $[12,21,25]$. Consequentially, the structure and quality of residency programs vary between different countries and teaching centers [12, 21]. In both professional societies as well as pertinent literature, there is clear evidence for a continued interest in optimizing neurosurgical residency $[15,24]$. To shine a light on the available options in neurosurgical education, this review will, in general, focus on resident education in Europe and the USA.

We are aware that comparing one single country, such as the USA, with the 47 countries in Europe is a challenging endeavor. This review, therefore, rather focuses on basic principles and does not provide in-depth analyses of every country or state. However, the authors would like to initiate a constructive discussion and are looking forward to comments from neurosurgical centers around the world regarding their neurosurgical training structure and options.

\section{Neurosurgical residency and training in Europe}

Looking at the literature, many proposals and guidelines have been published and recommended for neurosurgical training in Europe [17, 20, 23, 26]. The European Association of Neurosurgical Societies (EANS) [9] and the European Union of Medical Specialists (UEMS) [10] hold regular meetings with the goal of evaluating and improving neurosurgical education throughout Europe. The UEMS [10] is responsible to guarantee quality and improvement of medical specialties in Europe [23], whereas the EANS is an independent multinational association of both European neurosurgical societies and individual members [9]. Despite these efforts, it remains difficult to establish a consensus and find a commonly accepted concept for the education of European neurosurgical residents.

Although there is a comparable duration of 4 to 6 years of neurosurgical residency across Europe, the content thereof varies widely [24]. The reason for these diverse training conditions could be explained by (1) the number of sovereign countries in Europe (47 countries), (2) the unique historical educational concept in each of the European countries, and (3) the different socioeconomical setting of these countries [24]. Another cause can be found in the cultural autonomy of each country and hence the ability of each country's specific neurosurgical society to implement recommendations of the EANS for resident training [5] only to a varying degree. There rarely is any specific or mandatory neurosurgical curriculum for any teaching hospital. 


\section{Examination}

In the USA, the final board examination after neurosurgical residency is said to be competitive [25]. Although not yet fully implemented in every European country, a common final board examination was proposed by the EANS. This examination consists of two parts: the primary examination (written multiple choice questions) and the oral examination. They take place annually, since 1970, at the European neurosurgical training course [9]. Various European neurosurgical associations have their residents taking the annual EANS written board examination (primary examination) as well as a country-specific oral examination [22]. However, there are neurosurgical societies in Europe which do not require their neurosurgical candidates to take part in the final examination as proposed by the EANS. In contrast, those candidates are tested by means of state-specific oral examination only [7]. The written EANS exam consists of approximately 200 multiple choice questions in English covering neurosurgery, neuroanatomy, neuropathophysiology, neuropathology, neurology, neuroradiology, fundamental clinical skills, and other disciplines as deemed suitable and important. The examination takes $3 \mathrm{~h}$ and is administered annually [9]. The oral examination is a clinical problem-solving and patient management test. Case histories are given and candidates explain sequential steps in the management of the cases and the plan and performance of proposed operations, if indicated. The oral examination is held in English and consists of three parts ( $3 \mathrm{~h}$ in total), with about five to eight cases per hour. After passing the second exam, the candidate will be granted with the European Diploma in Neurosurgery; however, up to now, it does not constitute a license to practice neurosurgery in any European country [9].

\section{Content and research}

In Europe, the amount of research neurosurgical residents are required to complete varies widely. Although in most countries an educational plan is provided for the resident, research is not a compulsory part of residency. Different time frames for the implementation of a research component during training in Europe are possible. Post-MD research and MD-PhD programs are accessible in many academic institutions. Research can be done alongside the clinical work, within a temporary halt of the neurosurgical training or at the beginning or the end of the clinical work. In several countries, a 1-year break may be counted toward the required residency time. Established MD-PhD programs in many academic European training centers are a promising chance to integrate research with neurosurgical clinical training at an early point in education, and most commonly, the $\mathrm{PhD}$ is completed before starting a residency or early after beginning it.

\section{Work hours}

Since 2006, the European work time directive (EWTD) [8] has clearly mandated acceptable work hours for medical staff [24]. The weekly load should not exceed $48 \mathrm{~h}$. Some exceptions in countries with delayed implementation of the latter guidelines do exist. A comprehensive but controversial discussion can be found within the new EWTD [3, 19].

\section{Neurosurgical residency and training in the United States of America}

There is no standardized neurosurgical curriculum in the USA, although the proposal for a neurosurgical residency program as outlined by the American Board of Neurological Surgery (ABNS) [2] is commonly put into practice. Martin and Burn [17] reported that a representative US curriculum in neurosurgery at the most competent centers is well structured and routinely contains 1 or 2 years of research. The aim of a comprehensive neuroscience education is not only to turn out residents for future clinical demands and to prepare them for work the operating room but also to educate the next generation of 
neurosurgeons for competitive and state-of-the-art scientific demands. Residents who have undergone such an academic education during their residency are found to be heavily recruited from the public as well as from private institutions. The very important scientific part of the education in the USA favors the academic thinking and prepares the trainees for future leading positions in the academic neurosurgical settings.

\section{Neurological surgery training in general}

A general clinical surgical internship of 12 months should be completed before starting the residency and must be completed prior to beginning the third year of residency training [2]. Each resident must complete a minimum of 60 months of training as full-time resident in an Accreditation Council for Graduate Medical Education (ACGME) [1] accredited neurosurgical training program. The years are called post-graduate years (PGY), and contents are based on a well-planned curriculum [13, 16, 18]. At least 36 months must be devoted to a core of clinical neurosurgery with progressive responsibilities culminating in the last 12 months as most senior resident. At least 24 months of training in core clinical neurosurgery must be obtained in one institution [2]. The remaining 24 months must be devoted to aspects of the basic or clinical neurological sciences, which may include neuropathology, neuroradiology, and research.

As pre-structured and rather rigid as those guidelines may appear, there is the option for the program director to formulate modifications to fulfill specific training goals for an individual resident, the so-called Special Considerations clause [2]. Prior to acceptance of a candidate for oral examination, the ABNS [2] requires a "pass" on a challenging written board examination as well as statement from his or her program director to the effect that the candidate meets all necessary criteria to enlist for the oral part of the exam, which is taken 2-3 years later to include longitudinal practice data.

\section{Examination}

Each applicant for certification must first successfully pass the written "Primary Examination for credit toward certification." This examination is prepared by the ABNS [2] and includes extensive material on fundamental clinical skills, critical care, neuroanatomy, neurobiology, neurology, neuropathology, neuropharmacology, neuroradiology, neurosurgery, and other relevant disciplines deemed suitable and appropriate by the Board [2]. The final ABNS Board Certification examination then consists of an oral part which lasts about $3 \mathrm{~h}$ and covers the diagnosis, management, and outcome of surgical and medical diseases of the nervous system. Notwithstanding, given the growing tendency toward subspecialization, the examination covers all of neurosurgery and questions from all aspects of the discipline must be answered. The primary thrust relates to clinical practice via a case history format, including symptoms, findings, and results of diagnostic tests. Work-up, differential diagnosis, and management are evaluated. Particular attention is given to relevant anatomy, pathology, and physiological mechanisms, as well as descriptions of how operations should be performed, if clinically indicated. The examination is structured to focus on problems which neurosurgeons may expect to encounter and manage in general practice [2]. Once the examinee passes this oral examination, he is fully credentialed.

\section{Work hours}

The ACGME [1] requirements regarding a limit of resident work hours to below 80 per week have been implemented since July 2003 [11]. In 2005, already over $92 \%$ of the centers were found to comply with those guidelines [6]. Although it remains unclear how well institutions control resident work hours in detail and how accurately residents report overtime, the workload of residents has significantly decreased since its inception. Of note is 
the concept that taking calls from home is not considered to add to the $80 \mathrm{~h}$ of officially permitted workload of the week.

\section{Women in Neurosurgery [27]}

In the USA, compared to Europe, the development of the female role in medicine at large as well as in the field of neurosurgery is better documented and analyzed, but nonetheless far from being better established. The number of women in 2008 US neurosurgery remains notably below the $15 \%$ threshold required to achieve even "minority" status within a field [5]. The first woman obtaining neurosurgical board certification did so in 1960. The overall statistics in the USA reveal that only $179(3 \%)$ of the 5,854 ABNS [2] certified neurosurgeons are women.

Since 1970, the number of female medical students has steadily increased and finally outnumbered its male counterparts in 1995. A similar effect was not seen in the field of neurosurgery where we still find an ongoing disparity between the percentage of medical students, who are female, and the number of women entering neurosurgery training programs becoming ABNS-certified US neurosurgeons [5]. As discussed in the "white paper on the recruitment and retention of women in neurosurgery" [5], a presumed reason for the latter phenomenon is the lack of female role models and leaders in the field of neurosurgery as well as the lack of direct recruitment of female medical students during medical studies and internships. Women in Neurosurgery [27] has been active since 1989, having a women's advocacy and networking group. The aim of this organization is to attract women to the medical profession and to give recognition to female neurosurgeons who are bright, competent, and highly committed to the profession. In contrast to the USA, no similar networking group has been established in Europe.

\section{Discussion}

\section{Organization of curricula in general}

Although rules and guidelines by national neurosurgical parent organizations in the respective countries do exist, the different neurosurgical residency programs worldwide are independently organized by corresponding training centers. Exceptions from the mandated requirements for individual residents may be granted under the "special consideration" clause, and education can thus be modified individually. Therefore, despite the recommendations for a collective training in Europe [9] and strict guidelines by the ABNS [2] in the USA, an absolute and standardized curriculum has not been implemented. In general, neurosurgical residency curricula in the different States of the USA, when compared to Europe, tend to be more standardized, with a focus on well-structured monthly and yearly rotations based on the guidelines by the ABNS [2]. Each US ACGME-accredited neurosurgical institution has a designated residency program director overseeing the process of guideline implementation and quality control. This is no different in Europe, although not commonly established. However, there are a few centers in Europe having as structured neurosurgical training programs as counterparts in the USA. Beyond this, in the USA, the caseload of neuroendovascular procedures is shared between neurosurgery, interventional radiology, and neurology. Another neurosurgical subdivision in the USA having a multidisciplinary approach is radiosurgery. Due to the active involvement of neurosurgery within the latter two subdivisions $[12,21]$, their content is also reflected in the US residency training $[13,16,18]$. Since in Europe these subspecialties are rarely shared in a multidisciplinary approach, but rather belong solely to neuroradiology or radiation oncology, they are generally not part of neurosurgical training. 


\section{Research as a part of the residency}

In neurosurgery, clinical and basic research is an essential tool for residents and young surgeons to learn key concepts of existing therapy. Beyond that, it is essential to acquire necessary skills to be part of the evolution of science and to ultimately contribute and coshape future treatment strategies. Establishing and consolidating individual research interests and gaining expertise in a scientific field of interest offers the necessary skills to become a principal investigator. In the author's opinion, research expertise will become increasingly important in the competition for academic positions in neurosurgery. A major difference between Europe and the USA is the implementation of a 2-year research period in most of the US curricula, whereas in Europe and thus in most of the residency programs within the different countries in Europe, research activity is still considered as an additional and voluntary activity. However, given the 40- to 50-h week in Europe, there should be ample time to engage in ongoing research throughout the neurosurgical education, which obviously is more difficult to establish a more rigid organizational structure than in the American system. However, the US system allows for a period of undistracted and protected research time which creates the opportunity to participate in more "in-depth research activity," which often generates a body of substantial publishable data.

\section{Work hours}

In general, the work load (measured as the number of hours per week spent in the hospital) remains much higher in the USA ( $80 \mathrm{~h}$ in the USA compared to $40-50 \mathrm{~h}$ per week in Europe $[6,11]$. However, a considerable number of residents in Europe do take advantage of their non-clinical=out of hospital time (e.g., from overtime compensation), and this time is used to do clinical and/or basic/translational research. Obviously, less in-hospital time per week also means less neurosurgical operative or clinical teaching [6]. Indeed, the advantages for the US trainees stemming from work weeks in excess of $80 \mathrm{~h}$ are reflected in a consistently higher case load and thus faster acquisition of the required skills for successful completion of residency. It remains a matter of debate what a suitable case load during residency is to acquire those essential skills.

\section{Role of women in neurosurgery}

Neurosurgery as a field remains dominated by men, as the recently published white paper by Benzil et al. [27] demonstrates. Comparison of the percentage of female neurosurgeons practicing in the USA and Europe is currently not possible due to missing European data. On the other side, the female role in the professional activity of neurosurgery in the USA is well documented and remarkably reported below 15\% [5]. Unless substantial changes in work hours and in the content of the neurosurgical curriculum is not implemented, with the specific goal of creating programs favoring female role models as leaders in the field, a dramatic raise of the percentage of female neurosurgeons is not to be expected in the near future.

Although work hours in Europe would seem to facilitate integration of neurosurgery and family life, the truth is that academic neurosurgery remains one of the most time-consuming and competitive professions, just as it is in the USA. This is most likely due to the general perception that a certain amount of experience in a given time (=case load) is necessary to perform well in such a highly demanding profession, which has the life of others at hand. Further studies and better evaluation of women in neurosurgery in Europe are warranted.

\section{Conclusions}

The US residency programs overall tend to be more structured when compared to the corresponding curricula in Europe, although exceptions may apply. This is evident 
especially via the well-organized monthly and/or yearly rotations, which advance the resident during the consecutive PGYs [2]. Work hours for residents in most European countries remain limited to 40 to $50 \mathrm{~h}$ a week compared to at least $80 \mathrm{~h}$ per week in the USA. The lesser work load in Europe permits an ongoing research activity during several years of neurosurgical education. However, a 50-h work week also means lower case load, often leading to a longer acquisition time of the required skills to complete the residency. Choosing the right institution for the neurosurgical education has to be planned individually and should take into account different aims of the candidate. No general rule applies, but one does not necessarily need to go far to become a good surgeon. In case of pursuing an academic career, research at an early point, either within the MD-PhD program or as a postdoctoral candidate, is considered a must and facilitated in large academic institutions.

Integration of women into neurosurgery remains a major goal as proposed by the organization "Women in Neurosurgery" [27]. The lower rate of female residents compared to residents of other surgical disciplines remains striking and needs to be addressed in the future.

Cross talk between different neurosurgical centers is critical for a neurosurgical training, at present and much more in future, as globalization increases. For instance, currently over $90 \%$ of neurosurgery residency positions in the USA are held by american citizen [4]. There are no comparable data for Europe, but this tendency with country-specific residents seems to be present in European neurosurgical centers as well. A better standardization in the neurosurgical curriculum (worldwide and nationally) would facilitate the exchange of infield specific knowledge. It would also allow for a mutual exchange of personnel in such a fashion that national and international research collaborations will greatly profit. To create a more effective system of neurosurgical education worldwide, from our point of view, a multinational governing body might guide and even control education in neurosurgery, thereby granting excellent education through collaboration of residents and surgeons from a heterogeneous background.

\section{References}

1. Accreditation Council for Graduate Medical Education (ACGME). 2009. http://www.acgme.org

2. American Board of Neurological Surgery (ABNS). ABNS Board Certification. 2009. http:// www.abns.org/content/primary_certification_process.asp

3. Anwar M, Irfan S, Daly N, Amen F. EWTD has negative impact on training for surgeons. BMJ. 2005; 331(7530):1476. [PubMed: 16357004]

4. Barbieri RL, Anastos L, Michels KB. Economic factors and the percentage of residency positions filled by United States medical graduates. Obstet Gynecol. 2005; 106(3):581-584. [PubMed: 16135591]

5. Benzil DL, Abosch A, Germano I. WINS White Paper Committee et al . The future of neurosurgery: a white paper on the recruitment and retention of women in neurosurgery. J Neurosurg. 2008; 109(3):378-386. [PubMed: 18759565]

6. Cohen-Gadol AA, Piepgras DG, Krishnamurthy S, Fessler RD. Resident duty hours reform: results of a national survey of the program directors and residents in neurosurgery training programs. Neurosurgery. 2005; 56(2):398-403. discussion 398-403. [PubMed: 15670388]

7. Deutsche Gesellschaft für Neurochirurgie (DGNC). 2009. www.dgnc.de

8. Department of Health, UK. 2009. http://www.dh.gov.uk

9. European Association of Neurosurgical Societies (EANS). 2009. http://www.eans.org

10. European Union of Medical Specialists (UEMS). 2009. http://www.uems.net

11. Friedman WA. Resident duty hours in American neurosurgery. Neurosurgery. 2004; 54(4):925931. discussion 931-933. [PubMed: 15046659] 
12. Harbaugh RE, Agarwal A. Training residents in endovascular neurosurgery. Neurosurgery. 2006; 59(5 Suppl 3):S277-S281. discussion S3-13. [PubMed: 17053613]

13. Johns Hopkins University, Department of Neurosurgery. 2009. http://www.neuro.jhmi.edu

14. Long DM. Educating neurosurgeons for the 21st century. Neurosurg Q. 1996; 62(2):78-88.

15. Long DM. Competency-based training in neurosurgery: the next revolution in medical education. Surg Neurol. 2004; 61(1):5-14. discussion 14-25. [PubMed: 14706356]

16. Mayo Clinic, Department of Neurosurgery. 2009. http://www.mayoclinic.org

17. Martin M, Burn SC. Neurosurgical residency in the United States: a trainee's experience. Acta Neurochir (Wien). 2005; 147(11):1211-1212. discussion 1212. [PubMed: 16179964]

18. Massachusetts General Hospital, Department of Neurosurgery. 2009. http:// residents.neurosurgery.mgh.harvard.edu

19. Morris-Stiff GJ, Sarasin S, Edwards P, Lewis WG, Lewis MH. The European working time directive: one for all and all for one? Surgery. 2005; 137(3):293-297. [PubMed: 15746779]

20. Neil-Dwyer G, Lang DA, Trojanowski T. European Union of Medical Specialists. Neurosurgical training programme director position and responsibilities. Acta Neurochir Suppl. 2004; 90:13-16. [PubMed: 15553112]

21. Sauvageau E, Hopkins LN. Training in cerebrovascular disease: do we need to change the way we train residents? Neurosurgery. 2006; 59(5 Suppl 3):S282-S286. discussion S3-13. [PubMed: 17053614]

22. Schweizer Gesellschaft für Neurochirugie. 2009. http://www.swissneurosurgery.ch

23. Steers J, Reulen HJ, Lindsay KW. European Union of Medical Specialists, Joint Residency Advisory and Accreditation Committee. UEMS charter on training of medical specialists in the EU-the new neurosurgical training charter. Acta Neurochir Suppl. 2004; 90:3-11. [PubMed: 15553111]

24. Sure U, Miller D, Bozinov O. Neurosurgical training in Europe, problems and possible solutions. Surg Neurol. 2007; 67(6):626-628. discussion 628-33. [PubMed: 17512333]

25. Trojanowski T. Report of the JRAAC on the situation of the accreditation Training Programmes in Europe. Acta Neurochir (Wien). 2007; 149(12):1277-1279. [PubMed: 18075725]

26. Trojanowski T. Report of the JRAAC on the situation of the accreditation training programmes in Europe. Acta Neurochir (Wien). 2008; 150(8):851-853. [PubMed: 18651107]

27. Women in Neurosurgery (WINS). 2009. http://www.neurosurgerywins.org 